\title{
Gene-Environment-Time Interactions in Neurodegenerative Diseases: Hypotheses and Research Approaches
}

\author{
Walter G. Bradley ${ }^{a}$ Angeline S. Andrew ${ }^{b} \quad$ Bryan J. Traynor $^{c, d}$ Adriano Chiò $^{\mathrm{e}}$ \\ Tanya H. Butt ${ }^{\mathrm{b}}$ Elijah W. Stommel ${ }^{\mathrm{b}}$ \\ ${ }^{a}$ Department of Neurology, Miller School of Medicine, University of Miami, Miami, FL, USA; ${ }^{b}$ Department of \\ Neurology, Geisel School of Medicine at Dartmouth, Hanover, NH, USA; ' Neuromuscular Diseases Research \\ Section, Laboratory of Neurogenetics, National Institute of Aging, National Institutes of Health, Bethesda, MD, USA; \\ dDepartment of Neurology, Johns Hopkins University, Baltimore, MD, USA; e"Rita Levi Montalcini" Department of \\ Neuroscience, University of Turin, Turin, Italy
}

\section{Keywords}

Amyotrophic lateral sclerosis - Gene-environment-time interactions · Neurodegenerative diseases · Environmental risk factors · Genetic predisposition

\footnotetext{
Abstract

Background: Amyotrophic lateral sclerosis (ALS), Alzheimer's, and Parkinson's diseases are age-related neurodegenerative diseases. ALS is not a single entity but a syndrome with many different causes. In all 3 diseases, gene mutations account for only $10-15 \%$ of cases. Many environmental and lifestyle factors have been implicated as risk factors for ALS, though none have been proven to cause the disease. It is generally believed that ALS results from interactions between environmental risk factors and genetic predisposing factors. The advent of next-generation sequencing and recent advances in research into environmental risk factors offer the opportunity to investigate these interactions.
}

Summary: We propose a hypothesis to explain the syndrome of ALS based on the interaction of many individual environmental risk factors with many individual genetic predisposing factors. We hypothesize that there are many such combinations of individual, specific, genetic, and environmental factors, and that each combination can lead to the development of the syndrome of ALS. We also propose a hypothesis that explains the overlap between the age-related neurodegenerations and their genetic underpinnings. Age and duration of exposure are crucial factors in these age-related neurodegenerative diseases, and we consider how these may relate to gene-environment interactions. Key Messages: To date, genetic studies and environmental studies have investigated the causes of ALS separately. We argue that this univariate approach will not lead to discoveries of important gene-environment interactions. We propose new research approaches to investigating gene-environment interactions based on these hypotheses.

(c) 2018 S. Karger AG, Basel

\section{KARGER}

(c) 2018 S. Karger AG, Basel

E-Mail karger@karger.com

www.karger.com/aon
Walter G. Bradley, DM, FRCP

Department of Neurology, Miller School of Medicine

University of Miami, 1717 N. Bayshore Drive

Apt. 3134, Miami, FL 33132 (USA)

E-Mailw.bradley@miami.edu 


\section{Introduction}

Genes in Amyotrophic Lateral Sclerosis and Other Neurodegenerative Diseases

Since the discovery of mutations in SOD1 as a cause of familial amyotrophic lateral sclerosis (fALS) in 1993, neurogenetics research has progressively expanded the number of such genes that underlie ALS, to the point that we now know the genetic etiology of approximately twothirds of cases of fALS and roughly $10 \%$ of the sporadic form of the disease. Investigations of these mutations have identified common themes underlying ALS, such as disrupted RNA processing, autophagy, proteostasis and axonal transport. Many of these pathways are not specific for motor neurons, and hence it is unclear why they should produce a disease restricted to the motor nervous system.

To make matters even more complex, in age-related neurodegenerations, the same gene mutation can cause not only the ALS phenotype but also the clinic-pathological picture of seemingly distinct disease entities like Parkinson's disease (PD), Alzheimer's disease (AD), frontotemporal dementia (FTD), and progressive supranuclear palsy, as well as overlap syndromes. For instance, in fALS the same mutations of $V C P$ and C9orf 72 are present in individuals with ALS, FTD, and other neurological syndromes [1,2], and mutations in KIF5A have been variably associated with hereditary spastic paraplegia, CharcotMarie-Tooth disease, and infant myoclonic epilepsy, as well as ALS [3]. This phenomenon in genetics is termed pleiotropy, and it is remarkably common across neurodegenerative diseases. Two different scenarios add to this complexity. First, identical clinical entities can be caused by mutations in different genes (each affected individual carries a single mutation with a large effect on risk), and, second, polygenic inheritance where multiple variants across the genome work in unison to cause disease (each affected individual carries multiple small effect variants that cumulatively add up to result in disease) [4]. Such observations offer a clue to understanding the causes of these age-related neurodegenerative diseases, suggesting that other co-existent genetic or environmental factors direct the phenotypic pathway.

Environmental Toxins and Lifestyle as Risk Factors for $A L S$ and Other Neurodegenerative Diseases

The search for environmental causes of ALS has a history dating back to Kinnier Wilson's suggestion in 1907 that chronic lead exposure could cause the disease. There is now a long list of environmental and lifestyle factors, exposure to each of which has been incriminated as a risk factor for ALS. This means that exposure to each of these factors is present in a statistically significantly greater number of ALS patients than in control subjects. In environmental epidemiology, we describe each factor as being "associated with" ALS because it is difficult to prove that the factor causes the disease, since we cannot do placebocontrolled exposure studies in humans. In fALS, it is conceptually easier to accept that a specific gene mutation "causes" ALS because it is present in several affected members of the same family, and because we find the same mutation in other families with fALS.

Chronic exposure to many different environmental toxins and lifestyle factors has been associated with ALS, including heavy metals (lead and mercury); agricultural and industrial chemicals; cleaning solvents/degreasers; aromatic solvents; persistent organic pollutants; certain occupations; tobacco use; military service; and head injuries. Also, sporadic ALS and Guamanian ALS/parkinsonism/dementia complex (ALS/PDC) have been linked to exposure to cyanobacteria and to the neurotoxic cyanotoxin, $\beta$-N-methylamino-L-alanine (BMAA).

Dose is also important in regard to environmental toxins and lifestyle factors as potential causes of ALS. Are multiple minor head injuries equivalent to a single severe head injury? Is a large dose of a toxin in a short time more likely to cause a neurodegeneration than a small dose exposure over a longer time? Is there a particular period of life when exposure to a risk factor is more dangerous? Also, the term "pleiotropy," as described above for genetics, may be equally applied to the field of neurotoxicology, since several different diseases may be caused by a single toxin. For instance, chronic exposures to the same environmental factor, such as pesticides, head injuries or BMAA, have been linked to ALS, AD, and PD. Also, exposures to several toxins may be synergistic.

\section{Temporal Considerations in Exposures to Chronic Environmental Toxins}

Symptoms from exposure to a toxin can occur acutely within hours, sub-acutely within days or weeks, or after chronic exposure for years or decades. Wild introduced the term "exposome" to encompass the life-course of disease-related environmental exposures [5]. Of relevance to the present discussion, he also emphasized the difficulty of identifying the causative environmental and genetic factors, when both are at low penetrance in the population. The concept of interaction of gene-environmenttime (G-E-T) in ALS and neurodegenerative diseases is not new $[6,7]$. 
The enormous number of potentially harmful toxins and lifestyle factors to which humans are exposed over their lifetime helps explain the difficulty in establishing that any one toxin or lifestyle factor causes ALS. It is generally believed that diseases like cancer and neurodegenerations result from chronic exposure that extends over years or decades. Such exposures are likely to occur at a specific geographic location and during a certain time period. The concept of "time geography" was originally suggested by Hägerstrand [8] and expanded by Jacquez et al. [9] in ALS.

When investigating G-E-T interactions, we need not only to consider "time geography" but two other temporal issues. Is there a specific time period or epoch when such exposure is critical, such as before birth, during the period of brain development or during later life? Is the effect of the exposure simply cumulative or does the effect diminish with time after the exposure has stopped? These concepts must be incorporated into any attempt to investigate the latency of ALS resulting from environmental toxic exposures (i.e., the "incubation period"). One thing is certain however: studying environmental exposures solely at the time of symptom onset is unlikely to reveal all that needs to be known, since the neurodegenerative disease process probably began years prior to the onset of clinical symptoms. We have no empirical evidence regarding the "incubation period" of ALS, but work in cancer may be relevant, where, for instance, arsenic has been reported to increase the incidence of bladder cancer only after 10 years of exposure and the incidence continued to rise for 20 years after termination of exposure [10].

\section{The Role of Aging in the Pathogenesis of the}

\section{Neurodegenerative Diseases}

Time and aging must be taken into account in trying to understand the complex etiology of ALS, as well as the role of genetic and environmental risk factors. Hence, we need to speak of G-E-T interactions in the neurodegenerative diseases.

ALS is one of the age-related neurodegenerations, along with $\mathrm{AD}$ and $\mathrm{PD}$. In ALS, the age-specific rates increase progressively to about 75 and then drop [11, $12]$, while in $\mathrm{PD}$, the age-specific rates increase progressively to about 80 and drop in the oldest cohort [13, 14], and in $\mathrm{AD}$, the age-specific rates increase exponentially all the way to the oldest cohort $[15,16]$. The decrease in ALS might be due to failure to diagnose ALS in the old and frail but more likely indicates a more significant biological phenomenon that still remains to be understood. A similar change in phenotype and age-specific

G-E-T Interactions in Neurodegenerative Diseases rates occurred in the epidemic of Guamanian ALS/PDC (see below).

Theories of the cause(s) of aging abound. Discussions related to whether aging is genetically pre-programmed or whether it results from innate entropy causing the accumulation of stochastic episodes of degeneration that follow Gompertz' law continue. The seemingly finite number of divisions that somatic cells may undergo may be mirrored by a similar maximum number of episodes of transcription and translation that the DNA and RNA may undergo in post-mitotic cells like motor neurons. In that regard, it is worth noting that motor neurons are among the most metabolically active cells in the body. The high performance of this "Formula One engine" in large neurons over decades may make them inherently susceptible to environmental and aging processes. However, to say that ALS is due to premature aging of the motor system is to attempt to explain one enigma by another. It will be more productive to search for abnormalities of specific biological processes that precede motor neuron degeneration in ALS and the other age-related neurodegenerative diseases.

\section{Hypotheses Relating Gene-Environment Interactions in ALS and Other Neurodegenerative Diseases}

\section{Gene-Environment Interactions in ALS}

It is increasingly recognized that gene-environment interactions may underlie the syndrome of ALS [17]. Variations of more than 30 genes have been associated with sporadic ALS, but these variations have not been linked to specific environmental risk factors, though epigenetics is a new area that is ripe for investigation [18]. The alteration of gene expression through DNA methylation, histone modification, and non-coding RNA-associated gene silencing are all targets of environmental insults considered to induce and sustain epigenetic changes that might result in neurodegeneration. In that regard, advances in technology have made it feasible to assay methylation status across the genome in a robust, high throughput manner, allowing us to address this issue in a datadriven manner.

\section{How Many Causes of ALS Are There?}

ALS is phenotypically such a variable entity that someone unfamiliar with its diverse clinical progression and ultimate pathology might think that ALS comprises several different diseases. Attempts to classify different phenotypes as being separate diseases have been unsuccess- 


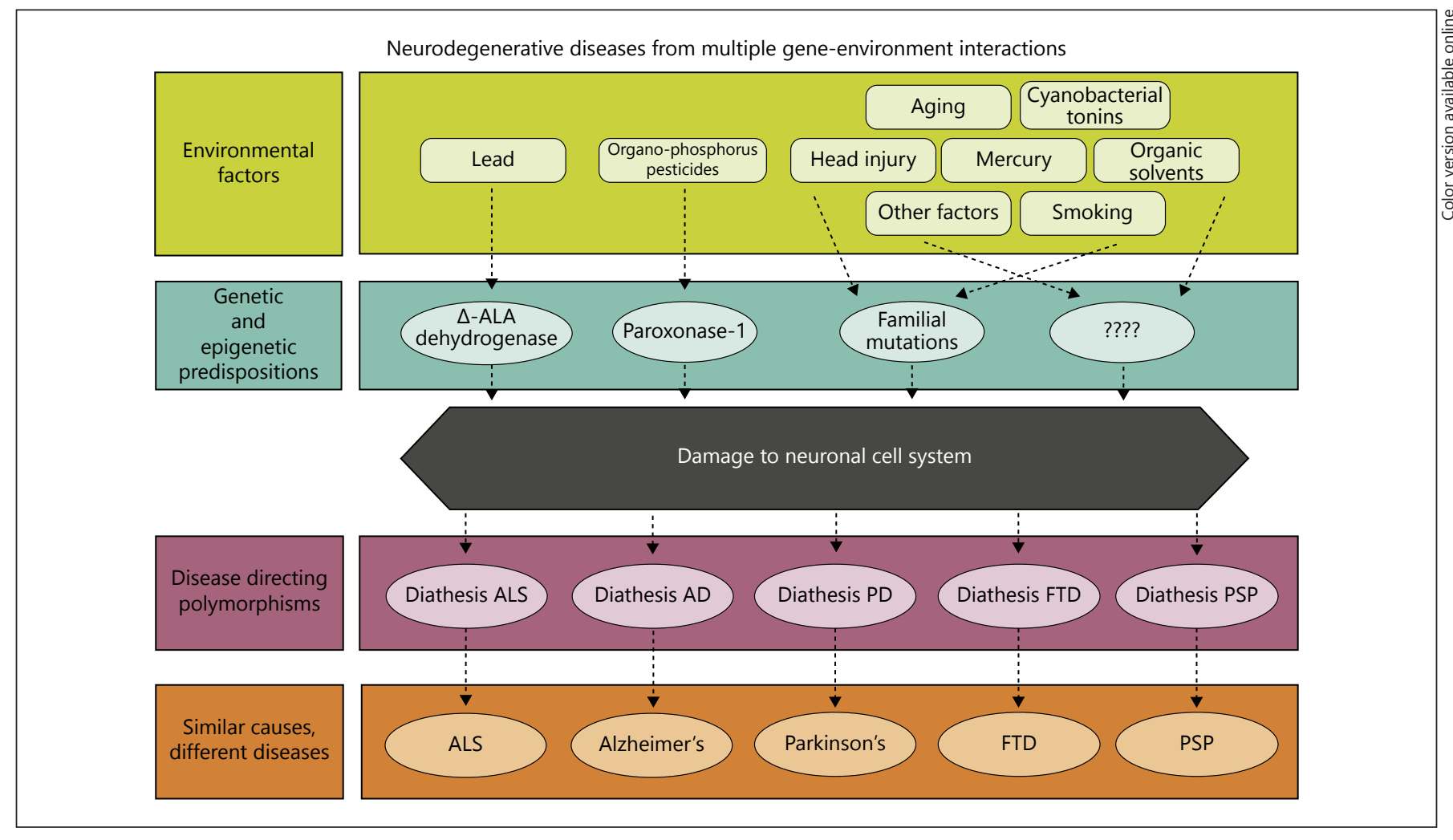

Fig. 1. Hypothetical pathways by which gene-environment interactions might lead to different disease phenotypes. Upper two panels: Hypothesis 1 - hypothetical gene-environment interactions. Lower two panels: Hypothesis 2 - hypothetical disease-directing polymorphisms leading to different disease phenotypes. ALS, amyotrophic lateral sclerosis; AD, Alzheimer's disease; PD, Parkinson's disease; FTD, frontotemporal dementia. ful, though the different phenotypes do have some prognostic significance $[19,20]$. Since the clinical picture, genetics, and neuropathology do not allow the separation of different underlying etiologies, we must look to the interactions of genes and environmental factors for the causes of the syndrome of ALS.

\section{Hypotheses about Gene-Environment-Time \\ Interactions in ALS}

To advance our understanding of what causes ALS, we must deal with the enormous complexity of G-E-T interactions. It is probable that there are hundreds of individual gene-environment interactions, each of which has its own particular time course and each of which is a separate cause of the syndrome of ALS. This concept leads to the hypothesis that each specific environmental factor interacts with a specific genetic factor - mutation, polymorphism or intronic DNA-based variation - to damage a specific metabolic pathway in motor neurons, thereby producing the clinic-patholog- ical phenotype of ALS (Hypothesis 1; Fig. 1: upper two panels). This hypothesis predicts that a population of ALS patients whose disease is caused by exposure to a certain environmental factor will have a genetic makeup that is different from that of a population of ALS patients whose disease is caused by exposure to another environmental factor, and that the difference will be in genes related to the metabolic response to that particular environmental agent. This hypothesis might also explain why in ALS clinical therapeutic trials a few patients appear to respond to the investigational drug, though the overall trial is negative.

Though we have no evidence to support this hypothesis at present, it suggests two parallel approaches to produce such empirical evidence: (1) Comparison of the genetic profile of a population of ALS patients with a high exposure to a specific environmental factor with that of a population of ALS patients without that high exposure; (2) Comparison of the exposome of a population of ALS patients that harbors a specific genetic risk factor with 
that of a population of ALS patients without that genetic risk factor.

This hypothesis might be thought to predict that each environmental risk factor affects a different metabolic pathway; but that may not be the case, since different environmental and genetic factors might affect multiple steps along the same metabolic pathway. Moreover, this hypothesis does not preclude that multiple environmental and genetic factors might interact synergistically to produce motor neuron damage.

A corollary to this hypothesis is that each gene mutation in fALS interacts with some combination of environmental factor, aging, and time in such a way as to trigger the disease. This would explain why after decades during which the mutation was present in the individual without causing symptoms, the disease suddenly appears. The key question is what happened in the exposome during the years before symptoms appeared? Perhaps it is a timelocked exposure to a specific environmental factor that leads to a critical accumulation of damage in motor neurons. G-E-T studies of twins discordant for ALS may be helpful to investigate this corollary.

How Can the Same Etiological Factor Produce Several Different Diseases?

The age-related neurodegenerative diseases, ALS, AD, PD, FTD, and progressive supranuclear palsy, have until recently been conceptualized as different diseases, despite being seen in different members of the same family with the same mutation (see above). ALS and FTD form a spectrum of disease, with a minority of patients existing in the wings with either pure ALS or pure FTD. Since we see pleiotropism both in genetics and in environmental toxicology, there must be some additional genetic or environmental factor in each such individual with a different phenotype that directs the neurodegenerative process into the different disease-defining pathways.

This concept leads to the following hypothesis: The clinic-pathological phenotype of disease that develops in an individual subjected to a variety of different gene-environment interactions depends on that individual's polygenic makeup (Hypothesis 2; Fig. 1: lower two panels). This hypothesis predicts that an individual who develops a disease phenotype will differ from another individual in the same family with the same mutation, who develops a different disease phenotype. This difference might be in exposures to different disease-related environmental factors, or in oligogenic or polygenic factors that either provide protection to or impose greater metabolic stress on one neuronal system than another.

G-E-T Interactions in Neurodegenerative Diseases

\section{Clues Regarding Pleiotropism from ALS/PDC}

in Guam

The epidemic of ALS/PDC in Guam is the clearest example of pleiotropism resulting from exposure to a single environmental toxin. In the 20 -year period following 1945 , the epidemic consisted only of ALS cases. In the following decades, the incidence of ALS decreased dramatically, and the phenotype gradually changed to predominantly parkinsonism and later to dementia [21, 22]. At the same time, the mean age of the patients affected by one of these neurodegenerative diseases gradually increased. The epidemic of ALS in Guam probably resulted from a time-related pulse in the exposure of the Chamorro natives. Guam ALS/PDC probably results from the Chamorro diet containing BMAA, produced by cyanobacteria in aerial roots of the cycad tree, which enters the food chain via the cycad fruit $[23,24]$. The pulse in dietary intake may have resulted from the period of nutritional deprivation during the Second World War and the availability of firearms after the war, which produced an increased intake of food items containing BMAA [25].

Burggren suggested that phenotypic drift might be due to a "washout" of epigenetic changes that might result from temporal changes in environmental exposures [26]. Perhaps some similar biological phenomenon underlies the differences in age-specific rates seen in the neurodegenerative diseases, ALS, PD, and AD (see above).

\section{Discussion}

\section{Empirical Approaches to Unraveling}

Gene-Environment-Time Interactions in the

Neurodegenerative Diseases

Studies to investigate Hypothesis 1 that ALS patients with a specific genetic predisposition to damage from an environmental factor will have a history of significantly greater exposure to that environmental factor than ALS patients without such exposure will require two different approaches as described above. On the genetic side, these studies will need to determine total genomic and epigenomic information in a large cohort of ALS patients and controls. Databases of more than 20,000 ALS patients and nearly 60,000 control subjects have been needed to reach our current knowledge of the genetic underpinning of ALS, but none of these studies has attempted to correlate their findings with the exposomes of the subjects.

On the environmental exposure side, the difficulties are even greater. In addition to the 30 or more environmental factors noted above that have been statistically 
linked to ALS, there are tens of thousands of chemicals and biological agents polluting our environment that might also be relevant. G-T-E studies will require the compilation of detailed environmental exposure databases (the exposome) of each one of the subjects in large casecontrol cohorts.

The estimation of exposures usually relies upon completion by subjects of questionnaires, which are generally qualitative and subject various levels of reliability. Geospatial analytical tools and satellite remote sensing are being increasingly used in environmental epidemiology of human diseases to estimate exposure to pollutants $[27,28]$.

We would be wrong to underestimate the sheer magnitude of this task [29]. The compilation of large cohorts of ALS patients and controls, where the temporal exposome of each subject has been recorded in detail, is something which is only just the beginning. Machine learning is likely to be invaluable in handling the otherwise daunting task of analyzing such mega data.

\section{Conclusions}

The hypotheses proposed in this paper suggest that research into G-E-T interactions in the non-familial forms of age-related neurodegenerations, such as ALS, should take two different directions. One should examine the genetic and epigenetic makeup of populations of ALS patients with high exposures to specific environmental factors, and to compare them to populations of ALS patients without such exposures. The other should compare the environmental exposure histories (the exposomes) of populations of ALS patients with and without a specific disease-related polymorphism or minor mutation. These studies will require biobanks of DNA samples from thousands of ALS patients and thousands of suitable controls with linked exposome data on each subject.

\section{Acknowledgment}

We thank Matthew Bough for preparing the figure and Dominic Facciponte for technical support.

\section{Disclosure Statement}

Dr. Walter G. Bradley has received grants from NIH (NINDS), the Centers for Disease Control and Prevention (ATSDR National ALS Registry), the ALS Association, the Muscular Dystrophy Association, and other foundations, and from nu-

merous pharmaceutical companies for participation in therapeutic trials and data safety monitoring committees. He was formerly a consultant for Gerson Lehrman Group. He is the chairman of the Advisory Committee of the Institute of Ethnomedicine for which he receives an honorarium. Dr. Elijah W. Stommel has received grants from the Centers for Disease Control and Prevention (ATSDR National ALS Registry), the ALS Association, and other foundations, and from pharmaceutical companies for participation in therapeutic trials. Dr. Angeline S. Andrew has received grants from NIH, the Centers for Disease Control and Prevention (ATSDR National ALS Registry), and the ALS Association. Dr. Bryan J. Traynor has received research grants from the Myasthenia Gravis Foundation, the Robert Packard Center for ALS Research, the ALS Association, the Italian Football Federation (FIGC), the Center for Disease Control and Prevention, the Muscular Dystrophy Association, Merck Inc, and Microsoft Research. Dr. Traynor is part of the Intramural Research Program at the National Institutes of Health and receives funding through the Intramural Research Program. Dr. Traynor holds patents on the clinical testing and therapeutic intervention for the hexanucleotide repeat expansion of C9orf72. Dr. Adriano Chiò has received grant from the European Union, the Italian Ministry of Health, and the Italian Ministry of Education, University and Research. He has received personal compensations for the participation to scientific boards by Mitsubishi Tanabe, Cytokinetics, Roche and Italfarmaco. T.H.B. has no conflicts of interest to declare.

\section{Authors Contribution}

W.G.B. developed the concepts, wrote the first draft of the manuscript, and developed the final paper. A.S.A. expanded the concepts and developed the final paper. B.J.T. expanded the concepts and developed the final paper. A.C. expanded the concepts and developed the final paper. T.H.B. collected data and developed the final paper. E.W.S. expanded the concepts and developed the final paper.

\section{References} family, one gene and three phenotypes: a novel VCP (valosin-containing protein) mutation associated with myopathy with rimmed vacuoles, amyotrophic lateral sclerosis and frontotemporal dementia. J Neurol Sci 2016; 368:352-358.

2 Nicholson AM, Rademakers R: What we know about TMEM106B in neurodegeneration. Acta Neuropathol 2016;132:639-651.

3 Nicolas A, Kenna KP, Renton AE, et al: Genome-wide analyses identify KIF5A as a novel ALS gene. Neuron 2018;97:1268-1283.e6.

4 Ali O: Genetics of type 2 diabetes. World j Diabetes 2013;4:114-123.

5 Wild CP: Complementing the genome with an "exposome": the outstanding challenge of environmental exposure measurement in molecular epidemiology. Cancer Epidemiol Biomarkers Prev 2005;14:1847-1850. 
6 Traynor BJ, Singleton AB: Nature versus nurture: death of a dogma, and the road ahead. Neuron 2010;68:196-200.

7 Al-Chalabi A, Hardiman O: The epidemiology of ALS: a conspiracy of genes, environment and time. Nat Rev Neurol 2013;9:617-628

8 Hägerstrand T: Reflections on "What About People in Regional Science?” Papers of the Regional Science Association 1989, vol 66, pp $1-6$.

9 Jacquez GM, Sabel CE, Shi C: Genetic GIScience: toward a place-based synthesis of the genome, exposome, and behavome. Ann Assoc Am Geogr 2015; 105:454-472.

10 Smith AH, Marshall G, Roh T, et al: Lung, bladder, and kidney cancer mortality 40 years after arsenic exposure reduction. J Natl Cancer Inst 2018;110:241-249.

11 Seals RM, Hansen J, Gredal O, et al: Age-period-cohort analysis of trends in amyotrophic lateral sclerosis in Denmark, 1970-2009. Am J Epidemiol 2013;178:1265-1271.

12 Henry KA, Fagliano J, Jordan HM, et al: Geographic variation of amyotrophic lateral sclerosis incidence in New Jersey, 2009-2011. Am J Epidemiol 2015;182:512-519.

13 Driver JA, Logroscino G, Gaziano JM, et al: Incidence and remaining lifetime risk of Parkinson disease in advanced age. Neurology 2009;72:432-438.

14 Savica R, Grossardt BR, Bower JH, et al: Incidence and time trends of drug-induced parkinsonism: a 30-year population-based study. Mov Disord 2017;32:227-234.
15 Kawas C, Gray S, Brookmeyer R, et al: Agespecific incidence rates of Alzheimer's disease: the baltimore longitudinal study of aging. Neurology 2000;54:2072-2077.

16 Vardarajan BN, Faber KM, Bird TD, et al: Age-specific incidence rates for dementia and Alzheimer disease in NIA-LOAD/NCRAD and EFIGA families: National Institute on Aging Genetics Initiative for Late-Onset Alzheimer Disease/National Cell Repository for Alzheimer Disease (NIA-LOAD/NCRAD) and Estudio Familiar de Influencia Genetica en Alzheimer (EFIGA). JAMA Neurol 2014; 71:315-323.

17 Al-Chalabi A, Calvo A, Chio A, et al: Analysis of amyotrophic lateral sclerosis as a multistep process: a population-based modelling study. Lancet Neurol 2014;13:1108-1113.

18 Li HF, Wu ZY: Genotype-phenotype correlations of amyotrophic lateral sclerosis. Transl Neurodegener 2016;5:3.

19 Chiò A, Mazzini L, D'Alfonso S, et al: The multistep hypothesis of ALS revisited: the role of genetic mutations. Neurology 2018; 91:e635-e642.

20 Meltz Steinberg K, Nicholas TJ, Koboldt DC, et al: Whole genome analyses reveal no pathogenetic single nucleotide or structural differences between monozygotic twins discordant for amyotrophic lateral sclerosis. Amyotroph Lateral Scler Frontotemporal Degener 2015; 16:385-392.

21 Román GC: Neuroepidemiology of amyotrophic lateral sclerosis: clues to aetiology and pathogenesis. J Neurol Neurosurg Psychiatry 1996;61:131-137.
22 Garutto RM: Lessons from the study of natural experiments of hyperdendemic foci of neurodegeneration; in Amyotrophic lateral sclerosis and the frontotemporal dementias. Chap 1, Oxford University Press 2012, pp 1-26.

23 Murch SJ, Cox PA, Banack SA. A mechanism for slow release of biomagnified cyanobacterial neurotoxins and neurodegenerative disease in Guam. Proc Natl Acad Sci U S A 2004; 101:12228-12231.

24 Murch SJ, Cox PA, Banack SA, et al: Occurrence of beta-methylamino-l-alanine (BMAA) in ALS/PDC patients from Guam. Acta Neurol Scand 2004;110:267-269.

25 Banack SA, Cox PA: Creating a simian model of guam ALS/PDC which reflects chamorro lifetime BMAA exposures. Neurotox Res 2018;33:24-32.

26 Burggren WW: Dynamics of epigenetic phenomena: intergenerational and intragenerational phenotype 'washout'. J Exp Biol 2015; 218(pt 1):80-87.

27 Shi X: A geocomputational process for characterizing the spatial pattern of lung cancer incidence in new hampshire. Annals of the Association of American Geographers 2009; 99:521-533.

28 Torbick N, Ziniti B, Stommel E, Linder E, Andrew A, Caller T, et al: Assessing cyanobacterial harmful algal blooms as risk factors for amyotrophic lateral sclerosis. Neurotox Res 2018;199-212.

29 Gibson G: Population genetics and GWAS: a primer. PLoS Biol 2018;16:e2005485. 